\title{
AUTOMATED CANDIDATE DETECTION FOR ADDITIVE MANUFACTURING: A FRAMEWORK PROPOSAL
}

\author{
Page, Thomas Daniel; Yang, Sheng; Zhao, Yaoyao Fiona
}

McGill University

\begin{abstract}
As additive manufacturing (AM) continues to grow in its abilities, so does the need for a quick and effective method of determining how it should be applied. Over time, these methods are naturally developed and passed on as tacit knowledge. However, with the rapid advancement of AM technologies, identifying parts which are eligible for AM as well as gaining insight on what value it may add to a product needs to be modelled in an objective and transferrable way. This paper presents a framework for determining the candidacy of a part or assembly for AM, represented by its economic feasibility and potential for AM-specific benefits. A set of selection criteria is developed with the goal of fast-screening in mind; that is specific data which can be automatically extracted from CAD models and resource planning databases. A case study is performed to validate the criteria and decision model chosen, as well as gain insight to the potential for a more widespread application. The decision model successfully identified economic feasibility and AM potentials, which suggests the results of the case study show promise for a semi-automatic decision support system for identifying AM candidates.
\end{abstract}

Keywords: Additive Manufacturing, Decision making, Machine learning, Selection criteria

\section{Contact:}

Page, Thomas Daniel

McGill University

Mechanical Engineering

Canada

thomas.page@mail.mcgill.ca

Cite this article: Page, T.D., Yang, S., Zhao, Y.F. (2019) 'Automated Candidate Detection for Additive Manufacturing: A Framework Proposal', in Proceedings of the 22nd International Conference on Engineering Design (ICED19), Delft, The Netherlands, 5-8 August 2019. DOI:10.1017/dsi.2019.72 


\section{INTRODUCTION}

As additive manufacturing (AM) continues to mature, so does the need for a quick and effective method of determining how it should be applied in product design. In the past, these methods are naturally developed and passed on as tacit knowledge. However, with the rapid advancement of AM technologies, identifying parts which are eligible for AM as well as gaining insight on what value it may add to a product needs to be modelled in an objective and transferrable way. This paper presents a framework for determining the candidacy of a part or assembly for AM, which has been developed based on its economic feasibility and potential for AM-specific benefits. A set of selection criteria is created with the goal of fast-screening in mind; that is specific data which can be automatically extracted from CAD models and resource planning databases. These selection criteria are used in conjunction with the candidacy framework to develop a semi-automated decision support system (DSS) for identifying candidates for AM. The ultimate goal of the DSS is to provide users with a quick and simple decision on whether their part, which is currently being manufactured via traditional methods, should be considered for AM. A case study is then performed which utilizes said DSS in the fast-screening of parts to determine where AM is economically feasible and where it may provide significant value-added potentials.

\section{BACKGROUND}

There are well defined rules for selecting a specific traditional manufacturing (TM) process (Lovatt and Shercliff, 1998), but making the choice between TM and AM is still a grey area for many organizations. Determining what makes a part eligible for AM is a relatively new area of research, and authors have taken a variety of approaches in finding the best method for evaluation. Because AM introduces new capabilities that are unknown to TM methods, AM breaks many of the established rules and guidelines developed for TM. While there are typically less restrictions on designers when utilizing AM, it is not simply a universal solution, and industries require support to know when and how AM should be applied. Therefore, comprehensive analysis of part candidacy for AM should not be limited to geometric considerations and should weigh the cost and value-added trade off.

Klahn et al. (2014) identified four criteria to determine product components eligible for re-design; these criteria are "integrated design", "individualization", "lightweight design", and "efficient design". While each of these are important considerations when assessing a product for AM potential, a more detailed breakdown of AM potentials can be found in the joint ISO/ASTM standard which outlines design guidelines for AM (ISO/ASTM, 2017). This method of criteria analysis focuses only on areas where AM will succeed, and fails to address what makes AM a poor choice for a specific part, therefore not rejecting parts with obvious limitations. Ultimately, the identification of a potential candidate for AM should be based on a more comprehensive assessment of the supply chain and other economic factors in addition to the potential benefits of AM.

Senvol outlined seven supply chain scenarios, as shown in Table 1, in which AM may be cost effective. These scenarios cover manufacturing expense, lead time, inventory costs, sourcing, remote operations, import/export costs, and functionality (Senvol LLC, n.d.). If a part falls into one or more of the scenarios, AM should be explored further, however, if a part fails to meet any of the scenarios, it will most likely not be cost effective. These scenarios provide an easy, high level way of checking for AM candidacy that allows companies to quickly sort through their part list. The benefit of this method is that the questions are easy to understand and can be quickly assessed; however, the focus of this research aims to determine the AM candidacy for parts in a more objective and efficient manner. Therefore, a mapping of this tacit knowledge into concrete data structures must be determined so that data can be collected and analyzed; for example, rather than simply asking if their import/export costs are high, asking for hard data will allow a DSS to consider the exact number along with all of the other criteria in forming a decision.

Lindemann and his colleagues (Reiher et al., 2013; Lindemann et al., 2015; Reiher et al., 2017) created a Trade-off Methodology (TOM) to help companies perform a quick, but more comprehensive, assessment of AM candidacy. The criteria was developed from industry input and domain expertise so to identify the most important factors of AM candidacy such as material consumption, processing time, economical aspects, size limitations, part complexity, part consolidation, and post processing. The TOM is filled out first by a company employee and then by an AM expert to evaluate the employee's responses and apply weightings to each criteria. Each criteria is weighted on a scale from 1-5, and while definitions of each rating are often provided, there is still a significant amount of subjectivity introduced 
Table 1. Seven scenarios where AM should be considered

\begin{tabular}{|l|l|}
\hline Scenario & Description \\
\hline Expensive to manufacture & $\begin{array}{l}\text { Do you have parts that are expensive because they are complex, have high fixed costs (e.g. } \\
\text { tooling), or are produced in low volumes? AM may be more cost effective. }\end{array}$ \\
\hline Long lead times & $\begin{array}{l}\text { Does it take too long to obtain certain parts? Are your downtime costs extremely high? Do } \\
\text { you want to increase speed to market? Using AM, you can often get parts more quickly. }\end{array}$ \\
\hline High inventory costs & $\begin{array}{l}\text { Do you overstock or understock? Do you struggle with long-tail or obsolete parts? AM can } \\
\text { allow for on-demand production, thus reducing inventory. }\end{array}$ \\
\hline Sole-sourced from suppliers & $\begin{array}{l}\text { Are any of your critical parts sole-sourced? This poses a supply chain risk. By qualifying } \\
\text { a part for AM, you will no longer by completely reliant on one supplier. }\end{array}$ \\
\hline Remote locations & $\begin{array}{l}\text { Do you operate in remote locations where it is difficult, time consuming, or expensive to } \\
\text { ship parts? AM may allow you to manufacture certain parts onsite. }\end{array}$ \\
\hline High import/export costs & $\begin{array}{l}\text { Do you pay substantial import/export costs on parts simply because of the location of your } \\
\text { business unit and/or your supplier? Onsite production by AM can eliminate these costs. }\end{array}$ \\
\hline Improved functionality & $\begin{array}{l}\text { With AM, it is possible to redesign a part to improve performance beyond what was } \\
\text { previously possible. }\end{array}$ \\
\hline
\end{tabular}

by the types of questions asked, as well as requiring an expert to analyze the questionnaire. This opens up the process to interpretation based on the expert's past experience and biases and the employee's idea of each criteria (Kruse et al., 2017). Allowing the decision to be formed based on the opinion of a single expert fails to provide the user with an objective result. Machine learning can be used to improve the subjectivity of their methods and create a more streamlined process. Reducing the amount of input required by an AM expert in the early stages allows their expertise to be saved for later on in the process when the decisions being made are less easily automated by a computer program.

Yao et al. (2017) introduced a hybrid machine learning recommendation system based on a design feature database to automatically associate parts with AM potentials. Design features were manually extracted based on engineering requirements from past instances and the targeted project. These studies are helpful in identifying parts for part-level AM applications such as lightweight and customization, but they fall short of handling assemblies. By restricting the system to not include assemblies, one of the largest AM potentials, part consolidation, can never be considered. This method also limits the amount of benefit to be gained by other potentials which are often used in conjunction with part consolidation, such as light-weighting.

Yang and Zhao (2018) proposed a new set of candidacy rules to support the identification of part candidates for part consolidation. The automation of the candidacy detection is automated in their following works Yang et al. $(2018 \mathrm{a}, \mathrm{b})$ with the consideration of modularization and cost. Their methods are specific for part consolidation potential and it requires a prerequisite of clearly defined mutual part relations which impose difficulties for large-scale products/systems. To generalize the part selection for all types of AM-enabled benefits and make the screening process more objective and cost-efficient, the work in this paper is developed. To generalize part selection for all types of AM-enabled benefits and make the screening process more objective and cost-efficient, an automated DSS is required to aid non-AM experts in finding part candidates before design for AM take place by AM experts.

\section{DEVELOPING THE CANDIDATE SELECTION FRAMEWORK}

A framework is proposed to enable more comprehensive, objective, and cost-efficient selection of part candidates for AM applications. The first and most important step in the framework is to derive the candidate selection criteria which can be used to identify parts to be considered for AM. A combination of criteria developed from TM metrics, AM metrics, and related literature are developed and filtered to determine if they meet the requirements for a fast-screening tool, this process is discussed further in the following section. Secondly, a database of parts and assemblies needs to be developed and each instanced needs to be assessed for AM potential and labelled according to the selection criteria. Then, when a new part or assembly needs to be assessed for AM candidacy, the same selection criteria will be extracted from it and fed to the decision model. The decision model will then use the labelled database of historical data to form the final AM candidacy decision. This process, as shown in Figure 1, can be repeated until all of the desired parts have been assessed. The process can be broken down into three major steps: the derivation of the selection criteria, the extraction and labelling of data to match the 
criteria, and forming the final decision via the DSS. This section of the paper will walk through the process shown in Figure 1 and explain how each step is carried out.

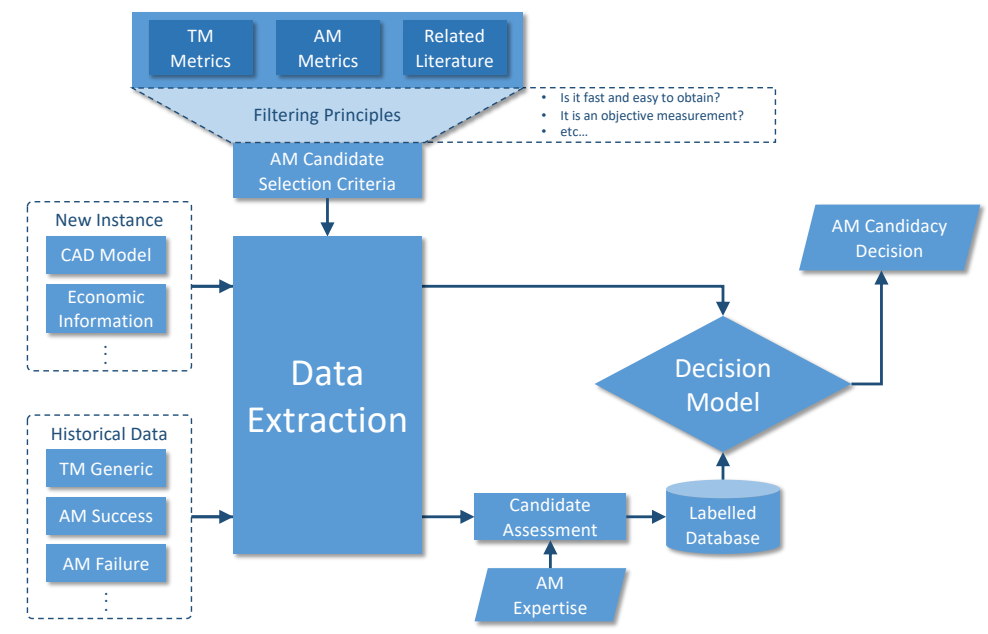

Figure 1. Candidate selection framework

\subsection{Derivation of candidate selection criteria}

The first step in developing the selection criteria was to perform a comprehensive analysis of factors which may affect the decision to manufacture a part using AM. These initial factors were formed based on a combination of both TM-specific metrics and AM-specific metrics.

Choosing criteria which are driven by data and not open to interpretation by the user are important for the DSS to remain unbiased. Criteria which are easily obtained through enterprise resource planning (ERP) databases and CAD drawings take highest priority as these can be mined quickly and further increase the amount of automation allowed by the DSS. Each category in the initial analysis of AM candidate selection (Figure 2) will now be examined to determine which are appropriate for a fast-screening AM DSS.

\subsubsection{Geometric complexity}

There is a significant difference between how TM and AM respond to geometric complexity, and it follows that this disparity makes the complexity of a part a good indicator of which manufacturing method should be used. With TM, as complexity increases, the manufacturing cost increases and manufacturability decreases. However, complexity has a much lesser effect on the cost of AM parts, and has led to the term "complexity for free" being used to describe this AM potential. Therefore, parts of great complexity should naturally be considered to have greater AM potential, as AM allows one to keep many of the complexities of a part without needing to sacrifice features for manufacturability. Given a design model, there are two methods for determining its complexity or manufacturability: feature-based and feature-free $(\mathrm{Li}, 2015)$. The geometric complexity criteria shown in Figure 2 very much represent the more traditional feature-based analysis. However, in this research the feature-free method is ultimately considered as it facilitates a quicker determination of complexity; all that is required is the part volume, part surface area, and bounding box volume. Valentan et al. (2008) found that a reasonable determination of complexity could be found using three different ratios: part volume to part surface area, bounding box volume to part surface area, and bounding box volume to part volume. Together these simple metrics give a good idea of geometric complexity, and can also be useful in determining the lightweighting potential of a part, as discussed in the next section. Another benefit of the feature-free method of complexity analysis is that the data required can either be automatically extracted from a design model, or when a design model is not available, manually entered by the user. The ease of obtaining these values allows all three to be used as a combined criteria for AM candidate detection. 


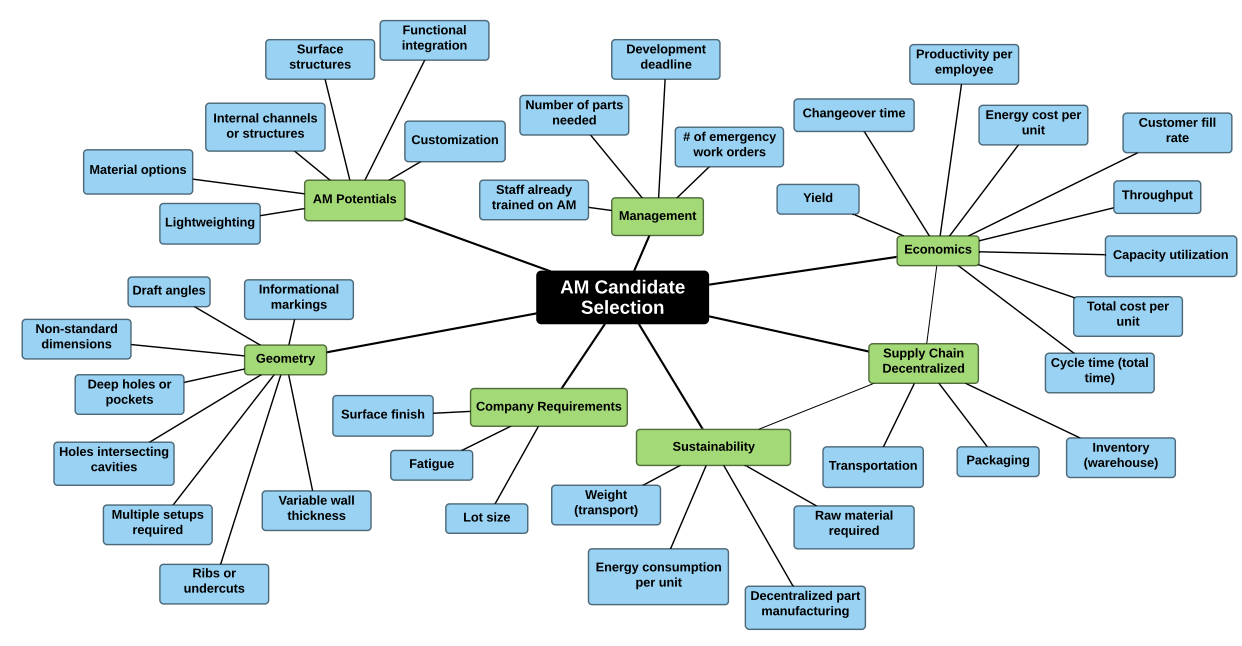

Figure 2. Initial analysis of AM candidate selection criteria

\subsubsection{AM potentials}

AM allows a unique set of value-added potentials which can often compensate for some of the higher costs associated with AM. These can be defined as "lightweight potential", "customization potential", "potential for internal channels/structures", "functional integration potential", "potential for designed surface structures", and "specific material options (hybrid materials, ...)" (ISO/ASTM, 2017). These potentials could be used as selection criteria for AM eligibility, since if a user wants to take advantage of an AM-specific potential, the likelihood that that part should be considered increases significantly. However, they were ultimately not chosen to be directly part of the criteria as the user operating the DSS need not be an AM expert and may not be familiar with AM potentials and where they can be applied. Instead, each individual potential is an output of the DSS, with a combination of automaticallyand user-completed questions in the criteria helping to form the decision. This facilitates the DSS not only being able to identify if printing the part seems economically feasible, but what AM potentials one should consider when redesigning the part.

A part that is optimized for TM will seldom see any economic benefits when directly switched AM, except in certain cases such as small batch size. The addition of these unique AM potentials allows the original part to achieve great enhancements when redesigned. The decision to use AM will ultimately depend on the trade-off between new or optimized features and product cost; something that is unique to each company and must be determined at a later stage in the decision process.

\subsubsection{Economical considerations}

The initial criteria in this section were largely derived from well-defined TM productivity metrics (Huang et al., 2002). However, once more abstract metrics were considered, such as those outlined in Table 1, the final criteria chosen became a hybrid of both TM and AM metrics. These criteria were selected as production costs, batch size, lead time, inventory costs, sole-sourced from suppliers, and import/export costs. TM metrics were chosen as they present potential weak spots for AM (e.g. batch size) and AM metrics were chosen which present potential benefits not accessible to TM (e.g. lower demand on inventory backlog).

\subsubsection{Supply chain \& sustainability}

Both the supply chain and sustainability categories were highly related to the economic considerations, and were therefore absorbed into the "economic analysis" category for the final criteria. For example, if the import/export costs of a TM part are very high, there would be a good potential for exploring a decentralized supply chain. Then, having more localized part manufacturing would decrease transportation and packaging costs as well as negative environmental effects. The questions in this category were difficult to answer, as the user of the DSS may not be familiar with how each may affect their part. By obtaining information about import/export and inventory costs, the questions in this category can be answered indirectly, and later be interpreted by the decision model. 


\subsubsection{Management \& company requirements}

The management section of the initial AM candidate selection criteria was designed to consider concerns such as staff knowledge of AM and development deadlines, and the company requirements section was designed to highlight areas where AM currently struggles when compared to TM such as surface finish and fatigue. Ultimately, the criteria from these sections were found to be ineffective in determining AM potential, or too abstract to effectively capture in an objective way. For example, while AM can improve time-to-market and lead to shorter development time (Muir and Haddud, 2018), the purpose of this screening is to find parts within a company which are already being manufactured through traditional methods which may have cost savings or other potentials when switched to AM. Development deadlines are much more of a concern for new designs and need not be considered in a quick screening process. With respect to the company requirements category, it was deemed ineffective to consider something like surface finish in the initial screening stage as there are both ways to achieve the desired properties through AM, and there is the potential that a redesign of the part or assembly could lead to the requirements being fulfilled in another way.

Table 2. Summary of part selection criteria for fast-screening of AM candidacy

\begin{tabular}{|c|c|c|}
\hline Selection Criteria & Units & Source \\
\hline \multicolumn{3}{|l|}{ Economic Analysis } \\
\hline Production costs & $\$ /$ part & ERP \\
\hline Batch size & \# of parts & ERP \\
\hline Inventory costs & $\$$ & ERP \\
\hline Import/export costs & $\$$ & ERP \\
\hline Sole-sourced from suppliers? & $\mathrm{Y} / \mathrm{N}$ & ERP \\
\hline \multicolumn{3}{|l|}{ AM Potentials Analysis } \\
\hline Does the part contain internal channels/structures? & $\mathrm{Y} / \mathrm{N}$ & User \\
\hline Would the part benefit from enhanced cooling? & $\mathrm{Y} / \mathrm{N}$ & User \\
\hline Number of components & \# & CAD \\
\hline Number of fasteners & \# & User \\
\hline Number of assembly interfaces & $\#$ & User \\
\hline Are there similar parts with slight modifications? & $\mathrm{Y} / \mathrm{N}$ & User \\
\hline Does the part require human body shape compliance? & $\mathrm{Y} / \mathrm{N}$ & User \\
\hline
\end{tabular}

\subsection{Extracting and labelling the data}

Expert analysis of as many traditionally manufactured parts as possible forms a knowledge base of the unique circumstances which give a part AM potential or lack thereof. Similar to how a human would learn and become an expert in any domain, the more exposure the DSS has to a variety of parts with differing manufacturing processes, sizes, shapes, complexities, etc., the more successful it will be in analyzing future parts. The experts analyze each part based on the developed selection criteria and make their decisions according to the list in Table 3. This labelled data can then serve as the input to the DSS.

Table 3. AM eligibility decision

\begin{tabular}{|l|}
\hline Candidacy Decision \\
\hline \multicolumn{1}{|c|}{ Economic Analysis } \\
\hline Economically feasible \\
\hline \multicolumn{1}{|c|}{ AM Potentials Analysis } \\
\hline Potential for lightweighting \\
\hline Potential for customization \\
\hline Potential for internal channels/structures \\
\hline Potential for part consolidation \\
\hline Potential for designed surface structures \\
\hline Potential for specific material options \\
\hline
\end{tabular}




\subsection{Choosing the decision model}

Machine learning is a promising solution to the problem of subjectivity in part selection as it can eliminate the need for experts to make the final decision on an individual part's potential for AM. Expert input is still required to create the database on which an algorithm forms its decision, but there are established methods which can be applied to reduce human bias in this data collection. Much of the industry input gathered is valuable and can be consolidated and altered to decrease subjectivity within this research. Current methods of evaluating a part's AM eligibility given a set of criteria either rely on AM experts to form the final decision, or a simple tally of the results to be compared with an acceptance threshold. As discussed, relying on AM experts is expensive and opens the door to the decision being biased by the expert's personal experience. On the other hand, while tallying results is less open to bias, it fails to capture the complex relationships between criteria. When provided with information-rich data that has been modelled in such a way to maximize domain knowledge, machine learning can perform very well in automatically finding the complex relationships that make up a model. For example, the Decision Tree algorithm forms a chain of if-else decisions to separate the data into classes; the deeper the tree, the more complex the decision model (Bishop, 2006). Then, the tree can be "pruned" to prevent overfitting on the training set. To ensure quality data, bias is even further reduced by designing the questions in such a way that there is seldom a subjective response. When a response must rely on a designer's opinion, the training set can be labelled by multiple designers to make sure the result is in agreement with the norm.

The model will return a list of parts which return true for any of the candidacy decisions shown in Table 3. Any positive response is returned because while a part may not economically feasible to be printed in its current state, it may see different results after being redesigned for AM. In other words, when a part shows no AM potential, it is disregarded; however, when it shows any AM potential, it is identified so that it can be analyzed by an expert in a more complete way. This method eliminated experts wasting time identifying parts which have been determined to show no AM potential.

\section{CASE STUDY}

To determine the feasibility of using machine learning to find AM part candidates, a preliminary case study is performed. In order to test the DSS, a database had to first be developed labelled according to the criteria described above in Table 2, then the machine learning model had to be trained on said data. When data from ERP databases or CAD drawings are unavailable, the user is asked to input them manually. The current work is focused on establishing the comprehensive set of criteria and test the feasibility of applying general ML models. Future research will be on finding more efficient and domain-specific ML models.

\subsection{Database structure}

The database contains approximately 120 training instances of varying parts and assemblies curated from various resources including parts from other projects at McGill University and online repositories like GrabCAD. An example entry is shown in Table 4.

\subsubsection{Missing data}

Much of the information required for the economic analysis must be obtained from protected ERP databases, and is inaccessible at this time. To mitigate this, the SolidWorks costing tool was used to generate a rough estimate for the cost of manufacturing each part via machining and then AM. Comparing these costs along with an estimated lead time is what formed the economic feasibility decision.

\subsection{Training}

Six different classifier algorithms from the sklearn python library (skl, n.d.) were trained on the database to determine which yielded the highest accuracy: Logistic Regression (LR), Linear Discriminant Analysis (LDA), K Nearest Neighbors (KNN), Decision Tree (DT), Naive Bayes (NB), and Support Vector Machine (SVM). These algorithms, as well as sklearn, are commonly known and widely used in the machine learning community. Detailed information on the mathematics behind each classifier can be 


\begin{tabular}{|c|c|c|c|c|c|}
\hline \multicolumn{6}{|l|}{ Image } \\
\hline \multicolumn{2}{|c|}{ Geometric } & \multicolumn{2}{|c|}{ Costs \& Economic Considerations } & \multicolumn{2}{|l|}{ Potential } \\
\hline Volume & $463.26 \mathrm{~cm}^{3}$ & Batch Size & 100 parts & Contains internal channels/structures? & No \\
\hline & $539.93 \mathrm{~cm}^{2}$ & Production & $\$ 212.48 /$ part & Would benefit from conformal cooling? & No \\
\hline BB (1) & $17.85 \mathrm{~cm}$ & Lead Time & 30 days & Number of components & 1 \\
\hline $\mathrm{BB}(\mathrm{w})$ & $11.10 \mathrm{~cm}$ & & & Number of fasteners & $\mathrm{N} / \mathrm{A}$ \\
\hline \multirow{3}{*}{$\mathrm{BB}(\mathrm{h})$} & $9.22 \mathrm{~cm}$ & & & Number of assembly interfaces & N/A \\
\hline & & & & Similar parts with slight modifications? & No \\
\hline & & & & Requires human body shape compliance? & No \\
\hline
\end{tabular}

found in a variety of introduction to ML texts (Hastie et al., 2001; Bishop, 2006). The algorithms mentioned can be considered the baseline for testing ML methods, and future development of this research will involve a more in depth assessment of the ML method chosen.

Given the training set of $120,10 \%$ of the samples were set aside for cross validation. The DT classifier consistently outperformed the others, scoring $>90 \%$ accuracy on all tests, and was therefore chosen as the classifier used for testing. This is likely due to its ability to capture complex relationships between criteria. An example of the training results is shown below in Table 5. In some tests, the classifiers reach $100 \%$ accuracy in training, testing, or both sets. A high testing score can be concerning as it suggests the model may be overfitting, however, the high accuracy in the test set suggests that this is not of issue. These extremely high scores may be caused by the training database not containing a diverse enough set of parts.

Table 5. ML Training Results

\begin{tabular}{|c|c|c|}
\hline Classifier & Training Set Accuracy & Test Set Accuracy \\
\hline LR & 0.9285 & 0.9285 \\
\hline LDA & 0.8809 & 0.9285 \\
\hline KNN & 0.8809 & 0.8571 \\
\hline DT & 1.0 & 1.0 \\
\hline NB & 0.8333 & 0.6428 \\
\hline SVM & 0.976 & 1.0 \\
\hline
\end{tabular}

\subsection{Testing}

Using the model generated from the DT classifier, a test part was evaluated that had not previously been seen by the algorithm. The part chosen was from GE's "Airplane Bearing Bracket Challenge" conducted through GrabCAD. The purpose of the challenge was to redesign the airplane bearing bracket for AM focusing on weight and strength improvements. This part was chosen as it is a clear example of a part that should be further considered for AM, and the machine learning algorithm should therefore be able to identify that.

The results of the DSS are summarized in Table 7. The classifier model found that the airplane bearing bracket would not be economically feasible given the current design, batch size, production cost, and lead time. However, it did find that there is a lightweighting potential for the part. Therefore, while the part is not economically feasible to print as-is, with AM reducing the raw material required to create it and furthermore the costs associated with running, the part very well may be worth switching to AM after being redesigned. Therefore, this part would be recommended to undergo a more thorough analysis for AM. 


\begin{tabular}{|c|c|c|c|c|c|}
\hline \multicolumn{6}{|l|}{ Image } \\
\hline \multicolumn{2}{|c|}{ Geometric } & \multicolumn{2}{|c|}{ Costs \& Economic Considerations } & \multicolumn{2}{|l|}{ Potential } \\
\hline Volume & $109.67 \mathrm{~cm}^{3}$ & Batch Size & 175 parts & Contains internal channels/structures? & No \\
\hline & $250.44 \mathrm{~cm}^{2}$ & Production & $\$ 37.98 /$ part & Would benefit from conformal cooling? & No \\
\hline BB (1) & $12.34 \mathrm{~cm}$ & Lead Time & 40 days & Number of components & 1 \\
\hline $\mathrm{BB}(\mathrm{w})$ & $8.46 \mathrm{~cm}$ & & & Number of fasteners & N/A \\
\hline \multirow[t]{3}{*}{$\mathrm{BB}(\mathrm{h})$} & $3.81 \mathrm{~cm}$ & & & Number of assembly interfaces & N/A \\
\hline & & & & Similar parts with slight modifications? & No \\
\hline & & & & Requires human body shape compliance? & No \\
\hline
\end{tabular}

Table 7. AM eligibility decision

\begin{tabular}{|l|c|}
\hline Output & Result \\
\hline Economically feasible & No \\
\hline Potential for lightweighting & Yes \\
\hline Potential for customization & No \\
\hline Potential for internal channels/structures & No \\
\hline Potential for part consolidation & No \\
\hline Potential for designed surface structures & No \\
\hline Potential for specific material options & No \\
\hline
\end{tabular}

\section{DIscussion}

The results presented in this paper show promise for a semi-automatic DSS for fast-screening of part candidates for AM, however, some things need to be considered as research progresses:

1. The database requires a more diverse set of parts to represent all of the AM potentials. Currently the most represented potential is lightweighting, with part consolidation in second. It follows that lightweighting and part consolidation consistently have the highest accuracy scores. Part consolation especially is seeing increased accuracy as the number of part consolidation-centric training examples increases, and this suggests that as more data becomes available for the other potentials their accuracy will increase as well.

2. The economic feasibility decision is currently solely based on estimations. However, with more complete data from an industry partner, the criteria presented in Table 2 should be sufficient in forming a good idea of economic feasibility. Obtaining this data and retesting for economic feasibility is a goal of future research.

Adding more data to the part database and adjusting the criteria and model to perform better is the main focus of future research. Currently the candidacy decisions are simply presented as yes or no; an interesting addition to this research would be to incorporate a tiered or scaled output where each individual candidacy decision is ranked (e.g. $90 \%$ lightweighting potential, low/medium/high lightweight potential, etc). This would allow users to specify the threshold at which they'd like to consider parts. For example, a company may be very new to AM and only wish to consider parts with a very high lightweighting potential; having a tiered candidacy decision would allow the DSS to filter out any parts that don't meet the specific threshold.

\section{CONCLUSIONS \& FUTURE WORK}

The AM candidate selection criteria presented in this paper provide an efficient way to quickly determine where and how AM should be applied within an organization. The case study performed shows how machine learning can be used to speed up the process while reducing bias in the final result. However, 
the case study could only be performed using a limited amount of data, as most of the criteria in the Economic Considerations section is sensitive and protected information. Without information from the ERP, the result can only be based on lead time and production cost. Future work in this area involves obtaining more complete data so that a true analysis of economic feasibility can be conducted.

\section{REFERENCES}

Bishop, C.M. Pattern Recognition and Machine Learning (Information Science and Statistics). Springer-Verlag, Berlin, Heidelberg, 2006. ISBN 0387310738.

Hastie, T., Tibshirani, R. and Friedman, J. The Elements of Statistical Learning. Springer Series in Statistics. Springer New York Inc., New York, NY, USA, 2001.

Huang, S.H., Dismukes, J.P., Shi, J., Su, Q., Wang, G., Razzak, M.A. and Robinson, D.E. "Manufacturing system modeling for productivity improvement”. Journal of Manufacturing Systems, Vol. 21 No. 4, pp. 249-259, 2002. ISSN 0278-6125. https://doi.org/10.1016/S0278-6125(02) 80165-0.

ISO/ASTM. Standard guidelines for design for additive manufacturing. Standard, ISO/ASTM, 2017.

Klahn, C., Leutenecker, B. and Meboldt, M. Design for additive manufacturing — supporting the substitution of components in series products. Procedia CIRP, Vol. 21, pp. 138-143, 2014. ISSN 2212-8271. https://doi.org/10.1016/j.procir.2014.03.145. 24th CIRP Design Conference.

Kruse, A., Reiher, T. and Koch, R. "Integrating am into existing companies - selection of existing parts for increase of acceptance". In Proceedings of the 28th Annual International Solid Freeform Fabrication Symposium, 2017.

Li, Y. Manufacturability analysis for non-feature-based objects. PhD thesis, Iowa State University, 2015.

Lindemann, C., Reiher, T., Jahnke, U. and Koch, R. "Towards a sustainable and economic selection of part candidates for additive manufacturing”. Rapid Prototyping Journal, Vol. 21 No. 2, pp. 216-227, 2015. https://doi.org/10.1108/RPJ-12-2014-0179.

Lovatt, A.M and Shercliff, H.R. "Manufacturing process selection in engineering design". part 1: the role of process selection. Materials \& Design, Vol. 19 No. 5, pp. 205-215, 1998. ISSN 0261-3069. https: //doi.org/10.1016/S0261-3069(98)00038-7.

Muir, M. and Haddud, A. "Additive manufacturing in the mechanical engineering and medical industries spare parts supply chain”. Journal of Manufacturing Technology Management, Vol. 29 No. 2, pp. 372-397, 2018. https://doi.org/10.1108/JMTM-01-2017-0004.

Reiher, T., Lindemann, C., Jahnke, U., Deppe, G. and Koch, R. "Holistic approach for industrializing am technology: from part selection to test and verification". Progress in Additive Manufacturing, Vol. 2 No. 1, pp. 43-55, Jun 2017. ISSN 2363-9520. https://doi.org/10.1007/s40964-017-0018-y.

Reiher, T., Lindemann, C., Moi, M. and Koch, R. "Impact and influence factors of additive manufacturing on product lifecycle costs". In Solid Freeform Fabrication Proceedings, 2013.

scikit (no date) scikit learn. Available at: https://scikit-learn.org/stable/ (Accessed: 23 February 2019).

Senvol LLC. Senvol LLC 7 scenarios table. http://senvol.com/additive-manufacturing/7-scenarios-table/, n.d. Accessed: 2018-10-09.

Valentan, B., Tomaz, B. and Drstvensek, I. "Basic solutions on shape complexity evaluation of stl data". Journal of Achievements in Materials and Manufacturing Engineering, Vol. 26, 012008.

Yang, S., Santoro, F., Sulthan, M.A. and Zhao, Y.F. "A numerical-based part consolidation candidate detection approach with modularization considerations". Research in Engineering Design, Oct 2018b. ISSN 1435-6066. https://doi.org/10.1007/s00163-018-0298-3.

Yang, S., Santoro, F. and Zhao, Y.F. "Towards a numerical approach of finding candidates for additive manufacturing-enabled part consolidation”. Journal of Mechanical Design, Vol. 140 No. 4: 041701-041701-13, Jan 2018a. ISSN 1050-0472. https://doi.org/10.1115/1.4038923.

Yang, S. and Zhao, Y.F. “Additive manufacturing-enabled part count reduction: A lifecycle perspective”. Journal of Mechanical Design, Vol. 140 No. 3: 031702-031702-12, Jan 2018. ISSN 1050-0472. https://doi.org/10.1115/1.4038922.

Yao, X., Ki Moon, S. and Bi, G. "A hybrid machine learning approach for additive manufacturing design feature recommendation”. Rapid Prototyping Journal, Vol. 23 No. 6, pp. 983-997, 2017.

https://doi.org/10.1108/RPJ-03-2016-0041.

\section{ACKNOWLEDGMENTS}

Financial support from the National Sciences and Engineering Research Council of Canada Discovery Grant RGPIN 436055-2013 is acknowledged with gratitude. 